\title{
Zur Erinnerung an Eckart Raubold
}

Am 5. Oktober 2010 starb Eckart Raubold nach längerer schwerer Krankheit im Alter von 72 Jahren. Prof. Dr. Raubold war promovierter Physiker und begann seine Laufbahn als Mitarbeiter und später Leiter des Rechenzentrums des Deutschen ElektronenSynchrotrons (DESY) in Hamburg. Dort gestaltete er bereits 1970 zusammen mit Prof. Lohrmann (Physik) den ersten Kurs "Einführung in die Informatik" einen Baustein für das Institut für Informatik an der Universität Hamburg. 1974 wechselte er als Institutsleiter zum Institut für Datenfernverarbeitung (vormals Deutsches Rechenzentrum DRZ) der Gesellschaft für Mathematik und Datenverarbeitung (GMD) nach Darmstadt.

Hier wurde dann bereits in den frühen 80iger Jahren unter Eckart Raubolds visionärer und charismatischer Führung der Samen für, Open Shops for Information Services (OSIS)' gelegt, die eine verbindliche elektronische Kommunikation in offenen ITSystemen (also auch zwischen bisher Unbekannten) ermöglichen sollte. Eckart Raubold verstand es, Mitarbeiter seines Institutes und externe Fachkollegen in Deutschland und Europa für diese Idee zu begeistern und so ein vom European Committee COST 11-bis gefördertes Projekt OSIS auf den Weg zu bringen. Die Ergebnisse lagen 1986 vor - wurden 1988 auf der CeBIT als Anwendungspilot präsentiert - und sind beeindruckend, weil ein so stabiles zukunftsweisendes Konzept selten ist:Viele Elemente - die Chipkarte als Sicherheitstoken, die asymmetrische Kryptographie, die vertrauenswürdige Dritte Instanz - sind enthalten, deren Ausgestaltung als nutzbringende anwendungsreife Lösungen heute noch die IT-Sicherheitsbranche prägen. Eckart Raubold hat diese Entwicklungen bereits sehr früh in einen gesellschaftlichen Kontext gestellt. Sein Verdienst ist es, die Notwendigkeit von neuen rechtlichen Rahmenbedingungen für die Anerkennung der Verbindlichkeit und für die damit verbundenen Voraussetzungen, während der Projektlaufzeit von OSIS erkannt und den ersten interdisziplinären Dialog mit Juristen befördert zu haben.

Die Akzeptanz von OSIS war bescheiden und zeigte auch, dass die Zeit für derartige Lösungen noch nicht reif war. Es spricht wiederum für Eckart Raubold, dass er dieses Signal als Herausforderung aufgegriffen hat und mit seinen Mitstreitern nun eine Institution anstrebte, die dauerhaft in die Gesellschaft hinein wirken, die Ergebnisse von OSIS ergänzen, und ihre Anwendung befördern sollte. Am 26. Januar 1989 trafen sich Vertreter von 14 Unternehmen und fassten den Beschluss, den Verein TeleTrusT Deutschland ins Leben zu rufen. Eckart Raubold wurde zum Vorstandsvorsitzenden bestellt und prägte die Arbeit von TeleTrusT bis 1994 maßgeblich. TeleTrusT konnte in den Folgejahren seine Anregungen und Visionen erfolgreich auf dem Weg in die technische, organisatorische und rechtliche Umsetzung begleiten.

Eckart Raubold erhielt 1989 für seine Forschungsarbeiten zum Themenbereich „Offene und sichere Kommunikation" den Forschungspreis Technische Kommunikation der Alcatel-Lucent Stiftung. Seit 1990 war er auch Honorarprofessor an der Universität Frankfurt. Von 1994 bis zum Ruhestand 2001 wirkte er als Forschungs- und Entwicklungsverantwortlicher bei der Deutschen Telekom.

Eckart Raubold hatte einen ausgewogenen Charakter. Was er sagte, war überlegt. Deshalb neigte man dazu, seine Meinung spontan zu akzeptieren. Das brachte ihm Anerkennung und verlieh ihm Charisma, auch wenn er dabei seine Person nicht in den Vordergrund stellte. Seine Meinung galt viel, nicht nur unter Fachkollegen, sondern auch in Politik und Verwaltung. Er versagte es sich aber, das auszunützen, wohl allein schon deshalb, weil seine Interessen - vom herkömmlichen Amateurfunk bis zur weiteren Evolution der Internetgesellschaft - bereits an die Informationstechnik vergeben waren. Diesbezüglich war er um nüchternen Realismus bemüht. Das tat dem Umstand dennoch keinen Abbruch, dass er oft als Lichtgestalt wahrgenommen wurde. Er blieb dabei bescheiden und eher reserviert. Wir trauern um ihn, können aber froh sein, dass es inn gegeben hat.

Helmut Reimer und Karl Rihaczek 